\title{
Single-cell Transcriptome Analysis Uncovers Heterogeneity and Key Regulators in Ibrutinib- resistant Chronic Lymphocytic Leukemia
}

Hui Jin

The First Affiliated Hospital of Nanjing Medical University

\section{Bin Huang}

Nanjing Medical University

\section{Zijuan Wu}

First Affiliated Hospital of Nanjing Medical University

\section{Huayuan Zhu}

The First Affiliated Hospital of Nanjing Medical University

\section{Hanning Tang}

the First Affiliated Hospital of Nanjing Medical University

Jiazhu Wu

The First Affiliated Hospital of Nanjing Medical University

\section{Danling Gu}

The First Affiliated Hospital of Nanjing Medical University

\section{Yun Cai}

Nanjing Medical University

\section{Luqiao Wang}

The First Affiliated Hospital of Nanjing Medical University

\section{Xueying Lu}

The First Affiliated Hospital of Nanjing Medical University

\section{Wei Xu}

The First Affiliated Hospital of Nanjing Medical University

\section{Kening Li}

Nanjing Medical University

\section{Qianghu Wang}

Nanjing Medical University

Jian-yong Li ( $\nabla$ lijianyonglm@126.com )

The First Affiliated Hospital of Nanjing Medical University

https://orcid.org/0000-0002-5878-945X

\section{Research}


Keywords: Chronic lymphocytic leukemia, Ibrutinib resistance, Single-cell RNA sequencing, LGALS1, LAG3

Posted Date: November 8th, 2021

DOI: https://doi.org/10.21203/rs.3.rs-998561/v1

License: (c) (1) This work is licensed under a Creative Commons Attribution 4.0 International License. Read Full License 


\section{Abstract}

\section{Background}

Ibrutinib as a widely used Bruton's tyrosine kinase inhibitor has shown outstanding value in clinical therapy for chronic lymphocytic leukemia (CLL). However, the bottleneck of ibrutinib resistance has caused widespread concerns, necessitating the exploration of novel targets.

\section{Methods}

Single-cell RNA sequencing (scRNA-seq) was used to characterize the heterogeneity of ibrutinib-sensitive (IBS) and -resistant (IBR) CLL patients and single-cell stemness estimation and metabolic pathway enrichment analysis were performed. Lectin galactoside-binding soluble 1 (LGALS1) and lymphocyteactivating gene 3 (LAG3) were screened as key factors by analyzing the RNA-sequencing data at bulk and single cell levels. Subsequently, pseudo-time trajectory analysis and gene set enrichment analysis were conducted. In addition, an IBR CLL cell line (MEC1-IR) was generated and RT-qPCR, western blotting, and immunofluorescence were performed to detect the expression of LGALS1 and LAG3. OTX008, a selective inhibitor of galectin-1 (Gal-1, encoded by LGALS1) was assessed in CLL cells and CCK8 and apoptotic assays were conducted for functional analysis.

\section{Results}

IBR CLL showed significantly different characteristics from IBS in terms of transcriptome expression and energy metabolism. LGALS1 and LAG3 were gradually upregulated in B cells along the evolution trajectory from IBS to IBR. Their expression was verified to be closely related to the prognosis of CLL, as well as sensitivity to ibrutinib. OTX008 could effectively suppress the proliferation and induce apoptosis of CLL cells, especially for those with ibrutinib resistance.

\section{Conclusions}

An LGALS1 and LAG3 gene panel is a promising indicator of ibrutinib resistance and a prognostic marker for CLL. OTX008 displays pronounced performance against CLL cells, especially with IBR, and might represent a novel therapeutic strategy for CLL.

\section{Background}

Chronic lymphocytic leukemia (CLL) involves leukemic cell infiltration and accumulation in lymphoid organs and is the most common leukemia in Western populations. Although its incidence is lower in China, the frequency of new cases is increasing $[1,2]$. The approval of Bruton's tyrosine kinase (BTK) inhibitors such as ibrutinib and acalabrutinib, and the B cell lymphoma-2 (Bcl-2) inhibitor venetoclax have revolutionized the treatment of CLL [3-7]. Whereas these novel agents alone or in combination induce long-lasting and deep remissions in most patients with CLL, drug resistance still occurs which is not well resolved. 
BTK, a key B cell receptor (BCR) signaling kinase, is amplified in CLL and activates proliferative and antiapoptotic signals [8]. Ibrutinib is an orally bioavailable BTK inhibitor that eliminates pro-survival pathway activation [9]. As the first FDA-approved BTK inhibitor, ibrutinib has shown significant clinical benefits for CLL patients [10]. However, current limitations including the development of resistance remain an unresolved clinical issue [11-13]. Mutations in BTK and PLCG2 are recognized as central factors for ibrutinib resistance, yet a low frequency of these mutations has been observed in our center [14-16]. Consequently, a comprehensive understanding of CLL clonal evolution driven by the selective pressure of drug therapy is vital for precise, individualized treatment.

Though next-generation sequencing $[17,18]$ has helped to clarify the key gene mutations, an understanding of the precise molecular mechanisms of ibrutinib resistance from a single cell perspective is needed, of which little information exists for CLL. Single-cell RNA sequencing (scRNA-seq) can assess gene expression in individual cells and systematically characterize heterogeneity at a higher resolution $[19,20]$. Moreover, this technology can be used to identify different cell subtypes based on relevant transcriptional modules [21, 22], and thus could be used to discover reliable biomarkers for clinical efficacy and drug-resistance.

Here, we characterized and analyzed the difference between CLL patients with ibrutinib-sensitive (IBS) and -resistant (IBR) by bulk and scRNA sequencing. IBR CLL exhibited unique characteristics in terms of transcriptome expression and energy metabolism. Seven distinct ibrutinib-resistant subpopulations were identified and two candidate genes, lectin galactoside-binding soluble 1 (LGALS1) and lymphocyteactivating gene 3 (LAG3) were screened, which were gradually upregulated in B cells along the evolution trajectory from IBS to IBR. LGALS1 is located on chromosome 22 and encodes galectin-1 (Gal-1), a protein involved in regulating physiological and pathological processes like cell transformation, proliferation, and adhesion, blood vessel formation, and immunosuppression [23]. LAG3 is an immune checkpoint molecule that was recently suggested as a novel therapeutic target in CLL [24-26]. A close correlation between LGALS1 and LAG3 expression was observed and these factors were found to be highly expressed in IBR CLL, with diagnostic and prognostic stratification, indicating that they might serve as biomarkers of IBR CLL. Moreover, OTX008 as a Gal-1 inhibitor suppressed proliferation and induced apoptosis in IBS and IBR cells. Together, we hope that these findings mighty offer a new therapeutic strategy for the treatment of IBR CLL.

\section{Methods}

\section{ScRNA-seq data processing}

ScRNA-seq libraries were sequenced from 150 bp 3' paired cDNAs using HiSeq Xten instruments. Raw data (BCL files) were converted to fastq files using bcl2fastq software (Illumina). Reads were aligned to a human genome reference (GRCh38) and a digital gene expression matrix built using the STAR algorithm in CellRanger (10x Genomics; v3.0.2). Quality control and further analyses were carried out using Seurat [27] (v3.1.5). To eliminate the effect of abnormal cells and technical noise on downstream analysis, we 
removed cells with abnormal gene counts or a high percentage of unique molecular identifiers (UMIs) mapped to mitochondria for each sample. Detailed clinical characteristics are showed in Table S1.

\section{ScRNA-seq sample integration and cell type recognition}

After log-normalization and variable feature identification based on variance stabilizing transformation, we integrated filtered cells from three IBR and four IBS CLL patients, as well as two healthy donors using "Findlntegration Anchors" and "IntegrateData" in Seurat with 30 dimensionalities [27], followed by scaling using "ScaleData". The statistical significance of principal component analysis scores was determined using "JackStraw" and dimension reduction was performed using the first 20 principal components. Data was visualized using T-distributed stochastic neighbor embedding (t-SNE) with "RunTSNE", whereas cell clusters were identified using "FindNeighbors" and "FindClusters" (resolution = 2). Differentially expressed genes (DEGs) were identified using the Wilcoxon Rank Sum test in "FindAllMarkers", with significance indicated by an average natural logarithm (fold change) $\geq 0.25$, Bonferroni-adjusted $p$-value $<0.05$, and minimum percentage $\geq 0.25$. Candidate markers were considered with CD14, CD1C, CD79A, $\mathrm{CD} 3 \mathrm{E}$, and KLRD1, and were used to annotate cell clusters. Single cell copy number variation (CNV) was estimated using inferCNV [28] (v0.8.2), with non-B cells as a reference.

Detailed materials and methods can be found in the supplemental data.

\section{Results}

\section{Singlecell landscape of PBMCs in patients with IBR and IBS CLL}

To study PBMC heterogeneity in CLL patients treated with ibrutinib and the underlying mechanism of ibrutinib resistance, we collected PBMCs from three IBR, four IBS CLL patients and two healthy donors and performed scRNA-seq using a droplet-based 10x Genomics platform. After quality control, we obtained 42,288 cells with a mean number of 4,760 cells per sample. The median number of unique molecular identifiers and genes per cell were 3,570 and 1,108 respectively. T-distributed stochastic neighbor embedding (t-SNE) visualization of filtered cells following integration and unsupervised clustering, revealed that cells from IBR and IBS samples were differently distributed in clusters, suggesting that IBR cells display a unique transcriptional pattern (Fig. 1A). To further differentiate these cells, we sorted them into 36 clusters (Fig. 1B) and annotated them as five major categories, specifically $\mathrm{CD} 79 \mathrm{~A}^{+} \mathrm{B}$-cells, CD3E ${ }^{+}$T-cells, KLRD1 ${ }^{+}$NK-cells, CD14 ${ }^{+}$monocytes, and CD1C ${ }^{+}$dendritic cells (Fig. $1 \mathrm{C}$, D; Fig. S1).

First, we calculated the proportion of cell types in each sample (Table S2). B cells exhibited significantly increased expansion in CLL patients compared to that in healthy donors. (Fig. 1E). As copy number variation (CNV) is a common phenomenon in CLL [29], we predicted InferCNV in IBR and IBS patients using scRNA-seq data Fig. 1F). We found that one IBR patient (R3) carried a deletion of the chromosome 8 short arm, consistent with the findings of Burger [30]. Two IBR patients (R2 and R3) carried a deletion of 
the chromosome 9 long arm and one IBR patient (R3) carried a deletion of the chromosome 4 short arm, implying that del( $9 q)$ and del( $4 p)$ might be associated with ibrutinib resistance. Obviously, we found that chromosome $6 p$ was often amplified, consistent with the results of Brown [31].

\section{B cells from IBR patients exhibit a unique transcriptional pattern}

Next, we investigated B-cell heterogeneity in IBR and IBS patients by re-clustering the 30,417 identified B cells into 21 clusters (Fig. 2A). B cells from IBR patients exhibited different distributions compared with those from IBS patients. CytoTRACE analysis revealed that B cells in IBR CLL patients had higher stem index scores, implying that abnormal $B$ cell stemness might be involved in the resistance to ibrutinib $(p<$ 2.2e-16, Fig. 2B and 2C).

According to the proportion of B cells from IBR samples, B-cell clusters were categorized into three main subgroups, namely IBR ( $>50 \%$ of B cells from IBR samples) and IBS ( $>70 \%$ of B cells from IBS or NC samples). Other clusters were defined as shared clusters and clusters with $<200$ cells were filtered (Fig. 2D). To elucidate the functional differences between IBR and IBS/shared clusters, we performed metabolic enrichment analysis. IBR clusters were significantly enriched in glycolysis and gluconeogenesis, which supply energy and support cancer cell growth (Fig. 2E, Table S3). We also found that many glycometabolism pathways, such as the pentose phosphate pathway, pentose and glucuronate interconversion, and fructose and mannose metabolism, were enriched in IBR clusters. Further, we found that glutathione metabolism was enriched in IBR clusters, consistent with Zhang who reported that elevated glutathione levels can increase leukemia cell survival and protect them against drug-induced cytotoxicity [32]. Together, these findings suggest that B cells from IBR patients exhibit a unique transcriptional and metabolic pattern compared with $B$ cells from IBS patients.

\section{Difference between intercellular interactions in the microenvironment of IBR and IBS cells}

Intercellular communication mediated by ligand-receptor complexes is essential for coordinating biological processes, such as differentiation and inflammation. Therefore, we studied the interaction between B cells and other types of cells from IBR and IBS clusters by performing cell-cell communication analysis using CellphoneDB. IBR-B cells displayed more interactions with monocytes, NK, T, and dendritic cells than IBS-B cells, suggesting that IBR-B cells could actively build connections with other cells to reshape the protective niche, which would be beneficial for cell survival (Fig. 2F). Previous studies have shown that co-culturing with stromal NK cells can increase the oxidative phosphorylation of CLL cells, promoting their proliferation [33]. Kurtova, et al. [34] also found that bone marrow stromal cells provide survival and drug resistance signals for CLL cells against spontaneous and drug-induced apoptosis.

Notably, the LGALS9-CD47 interactions only existed between T cells and IBR-B cells, and not IBS cells (Fig. 2G, Table S4), which has not previously reported in CLL. We also found that other B cell-T cell interactions such as MIF-TNFRSF14 and LILRA4-BST2 were specifically enriched in IBR clusters. These findings indicated that IBR B cells might reprogram intercellular interaction patterns in CLL patients. 
Therefore, the interaction between CLL cells and other microenvironment cells might be involved in the development of drug resistance.

\section{LGALS1 and LAG3 are associated with the transition between IBS and IBR}

Although genome-level features have been extensively studied in IBR patients with CLL [35, 36], the transcriptional characteristics are rarely reported. To improve our understanding of the underlying mechanism of ibrutinib resistance, we performed bulk RNA sequencing with PBMCs from 6 IBR and 40 IBS CLL patients. Ninety genes were significantly upregulated in the IBR group compared to levels in the IBS group, among which LGALS1, LAG3, and PTMS were the top three upregulated genes (Fig. 3A, Table S5). In addition, we identified highly expressed genes in each IBR cluster at the single cell level (719 genes; Table S6), including LGALS1, LAG3, and PTMS. Moreover, we investigated the developmental trajectory of B cells in IBR and IBS patients using pseudo-time analysis. Remarkably, B cells from IBR samples were distributed at the end of one state track, indicating that IBR cells might evolve from IBS cells (Fig. 3B). According to the pseudo-time analysis, we some genes were found to be gradually upregulated along the trajectory from IBS to IBR (Fig. 3C). LGALS1 was highly expressed in five single-cell IBR clusters (Fig. 3D). LGALS1 and LAG3 expression increased along the transition trajectory, whereas PTMS did not coincide with this trend (Fig. 3E). Interestingly, PTMS and LAG3 were found to be located adjacent to each other on chromosome 12p13.31 and bulk-RNA data indicated the co-expression of these genes.

LGALS1 and LAG3 typically act as immune checkpoints that repress innate and adaptive immune programs [37-39]. To further elucidate the function of LGALS1 and LAG3 in CLL patients, we analyzed the bulk RNA-seq data. Genes co-expressed with LGALS1 were identified, including S100A4, S100A6, and COX5B, which are associated with cancer cell invasion and proliferation [40] (Fig. 3F, Table S7), as well as genes co-expressed with LAG3, such as HSPG2, which is associated with poor prognosis in acute myeloid leukemia [42] (Fig. S2, Table S8).

GO analysis revealed that genes co-expressed with LGALS1 were significantly enriched in mitochondrial ATP synthesis-coupled electron transfer and oxidative phosphorylation (Fig. 3G, Table S9). LGALS1 might play an important role in the proliferation of CLL cells. In contrast, genes co-expressed with LAG3 were enriched for pathways related in glucose metabolism (Fig. S3). Together, LGALS1 and LAG3 are related to the metabolic pathways and might be involved in cell resistance to ibrutinib.

\section{High expression of LGALS1 and LAG3 closely correlates with poor outcome in CLL}

Next, we detected and analyzed the overall survival (OS) of CLL patients, finding that patients with higher LGALS1 and LAG3 expression showed poorer OS (Fig. 4A, left and middle). Moreover, Kaplan-Meier analysis demonstrated that patients with concurrent high expression of LGALS1 and LAG3 exhibited a worse OS compared to those with high LGALS1 or LAG3 expression respectively (Fig. 4A, right). To further validate the survival analysis results, we also analyzed GSE22762 data from the GEO database [43] 
which is comprise of $107 \mathrm{CLL}$ patient samples (Fig. 4B). As expected, these results both demonstrated that an LGALS1 and LAG3 gene panel is associated with poor prognosis for CLL patients.

To evaluate and confirm the clinical relevance of LAG3 and LGALS1 in CLL, we analyzed their expression and the clinical features of CLL patients (Fig. 4C). Patients with higher level of LAG3 and LGALS1 were found to have higher mutation rates for SF3B1 $(p=0.035)$ or NOTCH1 $(p=0.038)$. Immunoblots were then performed to compare protein levels between IBS and IBR patients, and the results revealed higher levels of Gal-1 and LAG3 in IBR patients (Fig. 4D, Fig. S4A). As Gal-1 is a secretory protein, we determined its plasma concentration as well as that of LAG3 in 16 treatment-naïve, refractory and/or relapsed (R/R) CLL patients using ELISA. As expected, Gal-1 and LAG3 levels were significantly higher in R/R CLL patients (Fig. S4B). Collectively, these results indicate that elevated expression of LGALS1 and LAG3 is associated with poor prognosis in CLL.

\section{LGALS1 and LAG3 are upregulated in the established ibrutinib-resistant cell line}

As LGALS1 and LAG3 indicated poor prognosis in CLL patients, we sought to explore the reasons for this with the acquired ibrutinib-resistant cell line (MEC1-IR, Fig. 5A). CCK8 assays indicated that MEC1-IR cells were significantly more resistant to ibrutinib than the parental cells (Fig. 5B). Annexin-V/PI staining also revealed a pronounced increase in cell death in MEC1 cells (Fig. 5C, D). Next, we examined the expression of LGALS1 and LAG3 in parental and resistant cells, revealing that they are markedly higher in MEC1-IR cells (Fig. 5E). Expectedly, immunoblotting and ELISA showed consistent results (Fig. 5F-H). These results demonstrate that LGALS1 and LAG3 are upregulated in IBR cells.

\section{The Gal-1 inhibitor OTX008 effectively inhibits the proliferation of CLL cells}

Recent studies have demonstrated the efficacy of OTX008, a Gal-1 inhibitor, in preclinical models of multiple tumors [44]. Therefore, we studied the anti-tumor effect of OTX008 on IBS and IBR CLL cells. First, we examined the expression of Gal-1 and LAG3 with OTX008 treatment. We found that OTX008 could decrease LAG3 mRNA levels (Fig. 6A). Meanwhile, the protein levels of Gal-1 and LAG3 were decreased in a dose-dependent manner in both CLL cell lines and primary cells (CLL-1) (Fig. 6B). This observation was validated by immunofluorescence (Fig. 6C). Though MEC1-IR cells displayed higher proliferation than MEC1 cells, the proliferative capacity of both groups was almost completely inhibited by OTX008 (Fig. 6D). Subsequently, flow cytometric analysis revealed that MEC1-IR cells underwent markedly increased apoptosis compared to that in MEC1 cells (Fig. 6E). Primary CLL cells were then treated with OTX008 and trypan blue staining revealed that OTX008 was more effective against the IBR group than IBS (Fig. 6F). Overall, our findings demonstrate the potential clinical utility of OTX008 for CLL, and particularly for IBR patients.

\section{Discussion}

CLL is a highly heterogeneous disease that can be treated using ibrutinib, a BTK inhibitor that has profound activity against this disease. However, the issue of ibrutinib-resistance has not been well solved 
yet. This study elucidated the transcriptional landscape of CLL patients using scRNA-seq. We identified IBR subpopulations and revealed their transcriptional and metabolic characteristics, and intercellular communication, finding that LGALS1 and LAG3 were upregulated in IBR primary cells and cell lines. LGALS1 and LAG3 expression was strongly associated with the prognosis of CLL patients and the panel of the two genes could be a potential biomarker of ibrutinib-sensitivity. Furthermore, the Gal- 1 inhibitor OTX008 induced apoptosis in and displayed particular efficacy against IBR CLL cells, indicating its potential clinical value for CLL.

Considerable evidence has shown that LGALS1 is often upregulated in tumor tissues [45] and correlates with tumor aggressiveness and treatment resistance [46]. Croci, et al. [47] indicated that Gal-1 contributes to the modulation of BCR signaling and is associated with poor outcomes in CLL. Here, we revealed that Gal-1 plays a critical role in CLL and might participate in the development of ibrutinib resistance. Moreover, OTX008 directly targeted Gal-1 to inhibit proliferation and promote apoptosis of CLL cells, especially with IBR-CLL cells. This could provide new treatment strategies to improve the clinical efficacy for patients with ibrutinib-resistant CLL.

ScRNA-seq also identified pronounced LAG3 expression in IBR clusters. Many studies have illustrated the role of LAG3 in immunotherapeutic regulation [48]. Dual PD1 and LAG3 immune checkpoint targeting has been shown to successfully control CLL development in preclinical mouse models [49]. According to the sequencing analysis and clinical relevance of Gal-1 and LAG3, we propose that a combination of OTX008 and anti-LAG3 monoclonal antibodies might achieve better efficacy for CLL, especially for IBR patients. However, further in vivo studies are required to determine the synergistic effect and toxicities. In addition, more clinical samples from patients with resistance to ibrutinib and other BTK inhibitors need to be collected.

Collectively, the present study comprises an in-depth analysis of the heterogeneity and microenvironment characteristics of IBR and IBS CLL. Our findings provide, for the first time, evidence that an LGALS1 and LAG3 gene panel could be used as an indicator of ibrutinib sensitivity, as well as a prognostic marker and potential therapeutic target for CLL patients. Further, we demonstrate that the Gal-1 inhibitor OTX008 could help to overcome ibrutinib-resistance, offering a promising combinatorial therapeutic strategy for IBR CLL patients.

\section{Conclusions}

Together, our findings demonstrate that IBR CLL cells exhibit a unique transcriptional pattern and indicate the prospect for future combination therapy. We propose that ibrutinib-resistance is mediated by LGALS1 and LAG3, two promising prognostic biomarkers and potential therapeutic targets in CLL, and that OTX008 might help to overcome the resistance to ibrutinib.

\section{Abbreviations}


BCR B cell receptor

BTK Bruton tyrosine kinase

CLL chronic lymphocytic leukemia

CNV copy number variation

DEGs differentially expressed genes

Gal-1 galectin-1

IBR ibrutinib-resistant

IBS ibrutinib-sensitive

LGALS1 lectin galactoside-binding soluble 1

LAG3 lymphocyte-activating gene 3

OS overall survival

PBMC peripheral blood mononuclear cell

scRNA-seq single-cell RNA sequencing

t-SNE T-distributed stochastic neighbor embedding

\section{Declarations}

\section{Ethics approval and consent to participate}

The human sample study was approved by the Medical Ethics Committee of First Affiliated Hospital of Nanjing Medical University. Written informed consent was obtained from all participants.

\section{Consent for publication}

Written informed consent was obtained from all patients.

\section{Availability of data and materials}

The datasets generated and/or analyzed during the current study are available in the GSA under accession number HRA000773.

\section{Competing interests}

The authors declare that they have no competing interests. 


\section{Funding}

This work was supported by grants from the National Natural Science Foundation of China (Grant \#81720108002, 81970146, 81770166, 81972358, 82170185 and 91959113), Key Research \& Development Program of Jiangsu Province (Grant \#BE2017733), Basic Research Program of Jiangsu Province (Grant \#BK20180036), Jiangsu Province's Medical Elite Programme (Grant \#ZDRCA2016022), Project of National Key Clinical Specialty, Jiangsu Provincial Special Program of Medical Science (Grant \#BE2017751).

\section{Authors' contributions}

$\mathrm{HJ}, \mathrm{KNL}, \mathrm{ZJW}$, and HYZ designed and performed most of the experiments; $\mathrm{BH}, \mathrm{KNL}$ and $\mathrm{YC}$ performed bioinformatics analysis of scRNA-seq and bulk RNA-seq data; HYZ, HNT and DLG collected the clinical samples; ZJW, JZW, LQW, and XYL collected and analyzed the clinical data; $\mathrm{HJ}, \mathrm{BH}$, and ZJW drafted the manuscript; $H J, K N L, Q H W$, and JYL critically revised the manuscript; JYL, KNL, QHW, and WX supervised the project. All authors read and approved the final manuscript.

\section{References}

1. Yang S, Gale RP, Shi H, Liu Y, Lai Y, Lu J, Huang X: Is there an epidemic of chronic lymphocytic leukaemia (CLL) in China? Leukemia research 2018, 73:16-20.

2. Yang SM, Li JY, Gale RP, Huang XJ: The mystery of chronic lymphocytic leukemia (CLL): Why is it absent in Asians and what does this tell us about etiology, pathogenesis and biology? Blood reviews 2015, 29(3):205-213.

3. Flinn IW, Hillmen P, Montillo M, Nagy Z, Illés Á, Etienne G, Delgado J, Kuss BJ, Tam CS, Gasztonyi Z et al: The phase 3 DUO trial: duvelisib vs ofatumumab in relapsed and refractory CLL/SLL. Blood 2018, 132(23):2446-2455.

4. Sun C, Nierman P, Kendall EK, Cheung J, Gulrajani M, Herman SEM, Pleyer C, Ahn IE, StetlerStevenson $\mathrm{M}$, Yuan $\mathrm{CM}$ et al: Clinical and biological implications of target occupancy in CLL treated with the BTK inhibitor acalabrutinib. Blood 2020, 136(1):93-105.

5. Chanan-Khan A, Cramer P, Demirkan F, Fraser G, Silva RS, Grosicki S, Pristupa A, Janssens A, Mayer $J$, Bartlett NL et al: Ibrutinib combined with bendamustine and rituximab compared with placebo, bendamustine, and rituximab for previously treated chronic lymphocytic leukaemia or small lymphocytic lymphoma (HELIOS): a randomised, double-blind, phase 3 study. The Lancet Oncology 2016, 17(2):200-211.

6. Byrd JC, Harrington B, O'Brien S, Jones JA, Schuh A, Devereux S, Chaves J, Wierda WG, Awan FT, Brown JR et al: Acalabrutinib (ACP-196) in Relapsed Chronic Lymphocytic Leukemia. The New England journal of medicine 2016, 374(4):323-332.

7. Fischer K, Al-Sawaf O, Bahlo J, Fink AM, Tandon M, Dixon M, Robrecht S, Warburton S, Humphrey K, Samoylova $\mathrm{O}$ et al: Venetoclax and Obinutuzumab in Patients with CLL and Coexisting Conditions. 
The New England journal of medicine 2019, 380(23):2225-2236.

8. Burger JA, Chiorazzi N: B cell receptor signaling in chronic lymphocytic leukemia. Trends in immunology 2013, 34(12):592-601.

9. Byrd JC, Furman RR, Coutre SE, Flinn IW, Burger JA, Blum KA, Grant B, Sharman JP, Coleman M, Wierda WG et al: Targeting BTK with ibrutinib in relapsed chronic lymphocytic leukemia. The New England journal of medicine 2013, 369(1):32-42.

10. de Claro RA, McGinn KM, Verdun N, Lee SL, Chiu HJ, Saber H, Brower ME, Chang CJ, Pfuma E, Habtemariam B et al: FDA Approval: Ibrutinib for Patients with Previously Treated Mantle Cell Lymphoma and Previously Treated Chronic Lymphocytic Leukemia. Clinical cancer research : an official journal of the American Association for Cancer Research 2015, 21(16):3586-3590.

11. Barr PM, Brown JR, Hillmen P, O'Brien S, Barrientos JC, Reddy NM, Coutre S, Mulligan SP, Jaeger U, Furman RR et al: Impact of ibrutinib dose adherence on therapeutic efficacy in patients with previously treated CLL/SLL. Blood 2017, 129(19):2612-2615.

12. Burger JA, Tedeschi A, Barr PM, Robak T, Owen C, Ghia P, Bairey O, Hillmen P, Bartlett NL, Li J et al: Ibrutinib as Initial Therapy for Patients with Chronic Lymphocytic Leukemia. The New England journal of medicine 2015, 373(25):2425-2437.

13. Mato AR, Nabhan C, Thompson MC, Lamanna N, Brander DM, Hill B, Howlett C, Skarbnik A, Cheson $\mathrm{BD}$, Zent $\mathrm{C}$ et al: Toxicities and outcomes of 616 ibrutinib-treated patients in the United States: a realworld analysis. Haematologica 2018, 103(5):874-879.

14. Woyach JA, Ruppert AS, Guinn D, Lehman A, Blachly JS, Lozanski A, Heerema NA, Zhao W, Coleman $\mathrm{J}$, Jones D et al: BTK(C481S)-Mediated Resistance to Ibrutinib in Chronic Lymphocytic Leukemia. Journal of clinical oncology : official journal of the American Society of Clinical Oncology 2017, 35(13):1437-1443.

15. Woyach JA, Furman RR, Liu TM, Ozer HG, Zapatka M, Ruppert AS, Xue L, Li DH, Steggerda SM, Versele $\mathrm{M}$ et al: Resistance mechanisms for the Bruton's tyrosine kinase inhibitor ibrutinib. The New England journal of medicine 2014, 370(24):2286-2294.

16. Ahn IE, Underbayev C, Albitar A, Herman SE, Tian X, Maric I, Arthur DC, Wake L, Pittaluga S, Yuan $\mathrm{CM}$ et al: Clonal evolution leading to ibrutinib resistance in chronic lymphocytic leukemia. Blood 2017, 129(11):1469-1479.

17. Landau DA, Sun C, Rosebrock D, Herman SEM, Fein J, Sivina M, Underbayev C, Liu D, Hoellenriegel J, Ravichandran $S$ et al: The evolutionary landscape of chronic lymphocytic leukemia treated with ibrutinib targeted therapy. Nature communications 2017, 8(1):2185.

18. Raponi S, Del Giudice I, Marinelli M, Wang J, Cafforio L, llari C, Piciocchi A, Messina M, Bonina S, Tavolaro $S$ et al: Genetic landscape of ultra-stable chronic lymphocytic leukemia patients. Annals of oncology : official journal of the European Society for Medical Oncology 2018, 29(4):966-972.

19. Zhou Y, Jia E, Pan M, Zhao X, Ge Q: Encoding Method of Single-cell Spatial Transcriptomics Sequencing. International journal of biological sciences 2020, 16(14):2663-2674. 
20. Moffitt JR, Hao J, Wang G, Chen KH, Babcock HP, Zhuang X: High-throughput single-cell geneexpression profiling with multiplexed error-robust fluorescence in situ hybridization. Proceedings of the National Academy of Sciences of the United States of America 2016, 113(39):11046-11051.

21. Khan S, Kaihara KA: Single-Cell RNA-Sequencing of Peripheral Blood Mononuclear Cells with ddSEQ. Methods in molecular biology (Clifton, NJ) 2019, 1979:155-176.

22. Rai KR, Jain P: Chronic lymphocytic leukemia (CLL)-Then and now. American journal of hematology 2016, 91(3):330-340.

23. Ruffo E, Wu RC, Bruno TC, Workman CJ, Vignali DAA: Lymphocyte-activation gene 3 (LAG3): The next immune checkpoint receptor. Seminars in immunology 2019, 42:101305.

24. Xia M, Luo TY, Shi Y, Wang G, Tsui H, Harari D, Spaner DE: Effect of Ibrutinib on the IFN Response of Chronic Lymphocytic Leukemia Cells. Journal of immunology (Baltimore, Md : 1950) 2020, 205(10):2629-2639.

25. Shapiro M, Herishanu Y, Katz BZ, Dezorella N, Sun C, Kay S, Polliack A, Avivi I, Wiestner A, Perry C: Lymphocyte activation gene 3: a novel therapeutic target in chronic lymphocytic leukemia. Haematologica 2017, 102(5):874-882.

26. Kotaskova J, Tichy B, Trbusek M, Francova HS, Kabathova J, Malcikova J, Doubek M, Brychtova Y, Mayer J, Pospisilova S: High expression of lymphocyte-activation gene 3 (LAG3) in chronic lymphocytic leukemia cells is associated with unmutated immunoglobulin variable heavy chain region (IGHV) gene and reduced treatment-free survival. The Journal of molecular diagnostics : JMD 2010, 12(3):328-334.

27. Stuart T, Butler A, Hoffman P, Hafemeister C, Papalexi E, Mauck WM, 3rd, Hao Y, Stoeckius M, Smibert P, Satija R: Comprehensive Integration of Single-Cell Data. Cel/2019, 177(7):1888-1902.e1821.

28. Patel AP, Tirosh I, Trombetta JJ, Shalek AK, Gillespie SM, Wakimoto H, Cahill DP, Nahed BV, Curry WT, Martuza RL et al: Single-cell RNA-seq highlights intratumoral heterogeneity in primary glioblastoma. Science (New York, NY) 2014, 344(6190):1396-1401.

29. Gaidano G, Rossi D: The mutational landscape of chronic lymphocytic leukemia and its impact on prognosis and treatment. Hematology Am Soc Hematol Educ Program 2017, 2017(1):329-337.

30. Burger JA, Landau DA, Taylor-Weiner A, Bozic I, Zhang H, Sarosiek K, Wang L, Stewart C, Fan J, Hoellenriegel $\mathrm{J}$ et al: Clonal evolution in patients with chronic lymphocytic leukaemia developing resistance to BTK inhibition. Nat Commun 2016, 7:11589.

31. Brown JR, Tesar B, Hanna M, Ash M, Fernandes SM, Macconaill L, Hainz U, Longtine J, Wu CJ, Werner L et al: Amplification of 6p Associated with Familial CLL. Blood 2010, 116(21):1009-1009.

32. Zhang W, Trachootham D, Liu J, Chen G, Pelicano H, Garcia-Prieto C, Lu W, Burger JA, Croce CM, Plunkett $W$ et al: Stromal control of cystine metabolism promotes cancer cell survival in chronic lymphocytic leukaemia. Nat Cell Bio/2012, 14(3):276-286.

33. Vangapandu HV, Ayres ML, Bristow CA, Wierda WG, Keating MJ, Balakrishnan K, Stellrecht CM, Gandhi V: The Stromal Microenvironment Modulates Mitochondrial Oxidative Phosphorylation in Chronic Lymphocytic Leukemia Cells. Neoplasia (New York, NY) 2017, 19(10):762-771. 
34. Kurtova AV, Balakrishnan K, Chen R, Ding W, SchnabI S, Quiroga MP, Sivina M, Wierda WG, Estrov Z, Keating $\mathrm{MJ}$ et al: Diverse marrow stromal cells protect CLL cells from spontaneous and drug-induced apoptosis: development of a reliable and reproducible system to assess stromal cell adhesionmediated drug resistance. Blood 2009, 114(20):4441-4450.

35. Woyach JA, Johnson AJ: Targeted therapies in CLL: mechanisms of resistance and strategies for management. Blood 2015, 126(4):471-477.

36. Komarova NL, Burger JA, Wodarz D: Evolution of ibrutinib resistance in chronic lymphocytic leukemia (CLL). Proc Natl Acad Sci U S A 2014, 111(38):13906-13911.

37. Mendez-Huergo SP, Blidner AG, Rabinovich GA: Galectins: emerging regulatory checkpoints linking tumor immunity and angiogenesis. Curr Opin Immunol 2017, 45:8-15.

38. Do JS, Visperas A, Sanogo YO, Bechtel JJ, Dvorina N, Kim S, Jang E, Stohlman SA, Shen B, Fairchild $\mathrm{RL}$ et al: An IL-27/Lag3 axis enhances Foxp3+ regulatory T cell-suppressive function and therapeutic efficacy. Mucosal Immunol 2016, 9(1):137-145.

39. Cagnoni AJ, Giribaldi ML, Blidner AG, Cutine AM, Gatto SG, Morales RM, Salatino M, Abba MC, Croci DO, Mariño KV et al: Galectin-1 fosters an immunosuppressive microenvironment in colorectal cancer by reprogramming CD8(+) regulatory $\mathrm{T}$ cells. Proceedings of the National Academy of Sciences of the United States of America 2021, 118(21).

40. Helfman DM, Kim EJ, Lukanidin E, Grigorian M: The metastasis associated protein S100A4: role in tumour progression and metastasis. Br J Cancer 2005, 92(11):1955-1958.

41. Gao SP, Sun HF, Jiang HL, Li LD, Hu X, Xu XE, Jin W: Loss of COX5B inhibits proliferation and promotes senescence via mitochondrial dysfunction in breast cancer. Oncotarget 2015, 6(41):4336343374.

42. Zhou X, Liang S, Zhan Q, Yang L, Chi J, Wang L: HSPG2 overexpression independently predicts poor survival in patients with acute myeloid leukemia. Cell Death Dis 2020, 11(6):492.

43. Herold T, Jurinovic V, Metzeler KH, Boulesteix AL, Bergmann M, Seiler T, Mulaw M, Thoene S, Dufour $A, P a s a l i c Z$ et al: An eight-gene expression signature for the prediction of survival and time to treatment in chronic lymphocytic leukemia. Leukemia 2011, 25(10):1639-1645.

44. Astorgues-Xerri L, Riveiro ME, Tijeras-Raballand A, Serova M, Rabinovich GA, Bieche I, Vidaud M, de Gramont A, Martinet M, Cvitkovic E et al: OTX008, a selective small-molecule inhibitor of galectin-1, downregulates cancer cell proliferation, invasion and tumour angiogenesis. European journal of cancer (Oxford, England : 1990) 2014, 50(14):2463-2477.

45. Goud NS, Soukya PSL, Ghouse M, Komal D, Alvala R, Alvala M: Human Galectin-1 and Its Inhibitors: Privileged Target for Cancer and HIV. Mini reviews in medicinal chemistry 2019, 19(16):1369-1378.

46. Nambiar DK, Aguilera T, Cao H, Kwok S, Kong C, Bloomstein J, Wang Z, Rangan VS, Jiang D, von Eyben $\mathrm{R}$ et al: Galectin-1-driven T cell exclusion in the tumor endothelium promotes immunotherapy resistance. The Journal of clinical investigation 2019, 129(12):5553-5567.

47. Croci DO, Morande PE, Dergan-Dylon S, Borge M, Toscano MA, Stupirski JC, Bezares RF, Avalos JS, Narbaitz M, Gamberale R et al: Nurse-like cells control the activity of chronic lymphocytic leukemia B 
cells via galectin-1. Leukemia 2013, 27(6):1413-1416.

48. Gassner FJ, Zaborsky N, Catakovic K, Rebhandl S, Huemer M, Egle A, Hartmann TN, Greil R, Geisberger R: Chronic lymphocytic leukaemia induces an exhausted T cell phenotype in the TCL1 transgenic mouse model. British journal of haematology 2015, 170(4):515-522.

49. Wierz M, Pierson S, Guyonnet L, Viry E, Lequeux A, Oudin A, Niclou SP, Ollert M, Berchem G, Janji B et al: Dual PD1/LAG3 immune checkpoint blockade limits tumor development in a murine model of chronic lymphocytic leukemia. Blood 2018, 131(14):1617-1621.

\section{Supplemental Figures}

Supplemental Figures are not available with this version.

\section{Figures}


A

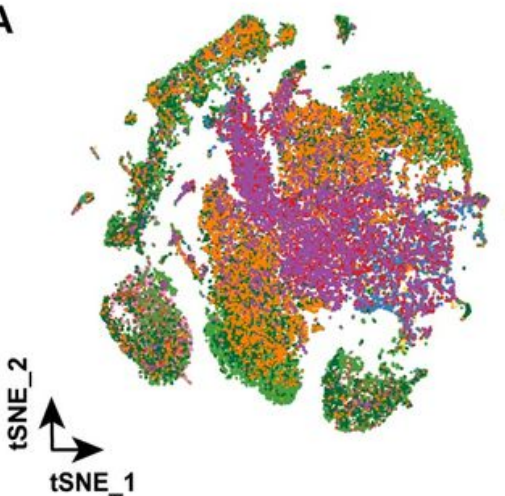

C
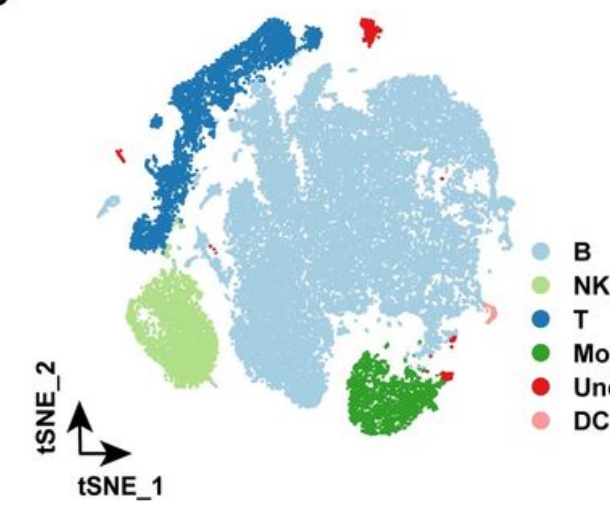

- NK

- $\mathrm{T}$

- Mono

- Unclassified

- DC

D

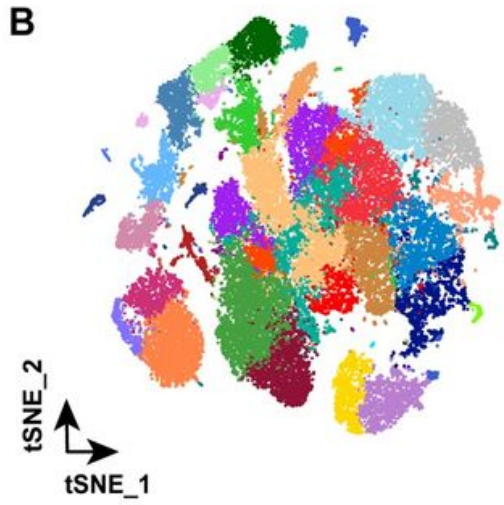

- $0 \quad 18$

$2: 20$

- $3: 21$

- 4 - 22

- 5 . 23

$\because 6: 24$

- $8: 26$

- 9.27

- 10.28

-11. 29

- 12 . 30

- $13: 31$

- 14.32

- $15 \cdot 33$

$16 \cdot 34$
$17: 35$
E

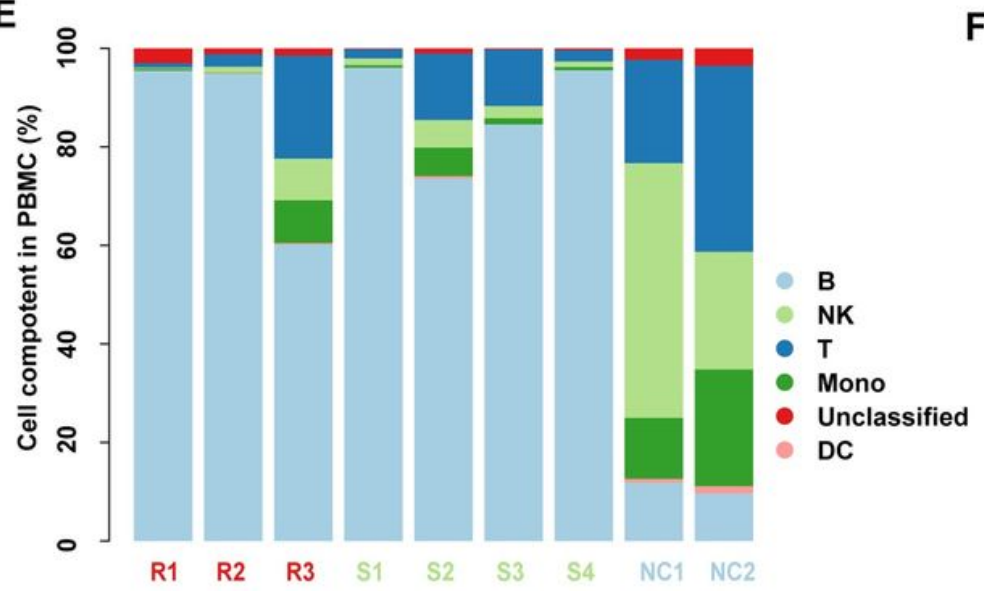

F
- Resistant

Sensitive

Normal

DC

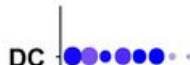

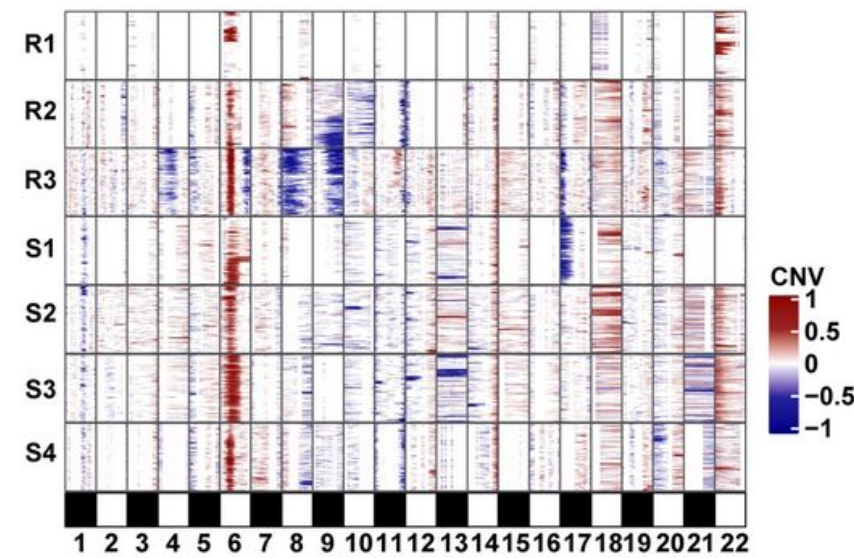

\section{Figure 1}

Expression profiling of 42288 single cells from the PBMC of nine samples. A T-SNE plot of all single cell data (10x Genomics). R1-3 represents three ibrutinib-resistant (IBR) cases. S1-4 represents four ibrutinibsensitive (IBS) cases. N1-2 represents two normal donors. B Result of the unsupervised clustering of all cells. C Cell-type annotation in the t-SNE plot. D Highly expressed genes in different cell types. Dot size indicates the gene expression percentage in the same cell type. Color indicates the average gene expression in the same cell type. E PBMC components in nine samples. F Large-scale copy number variation (CNV) in single cells inferred based on scRNA-seq data. 
A
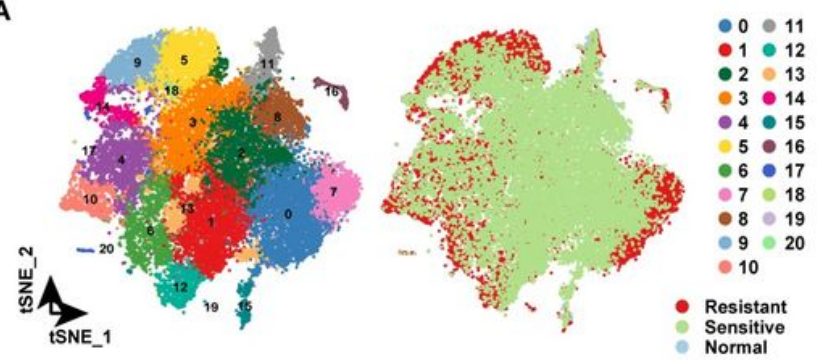

D

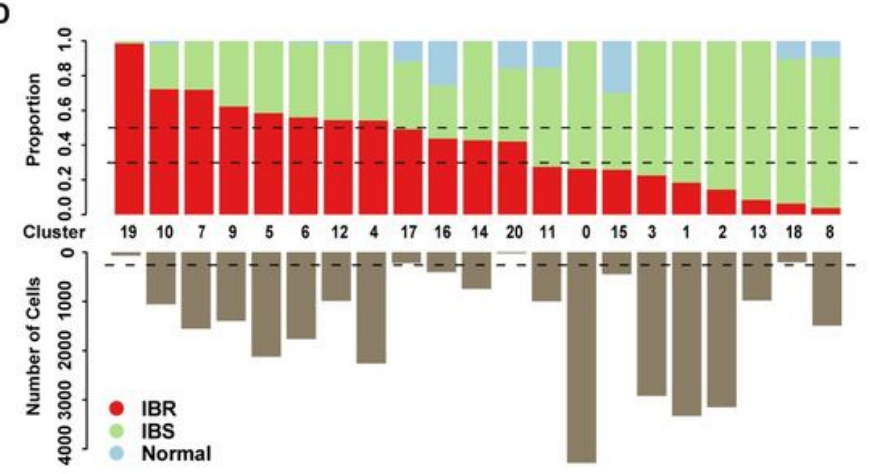

B
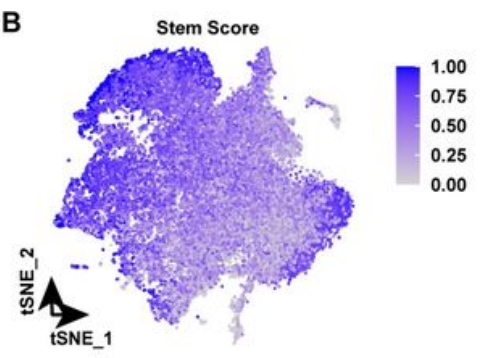

C

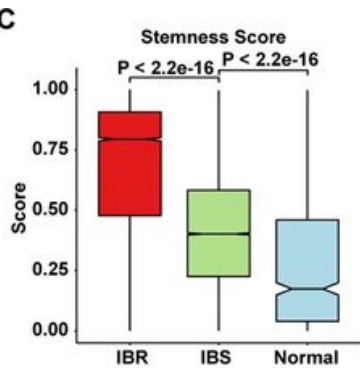

E

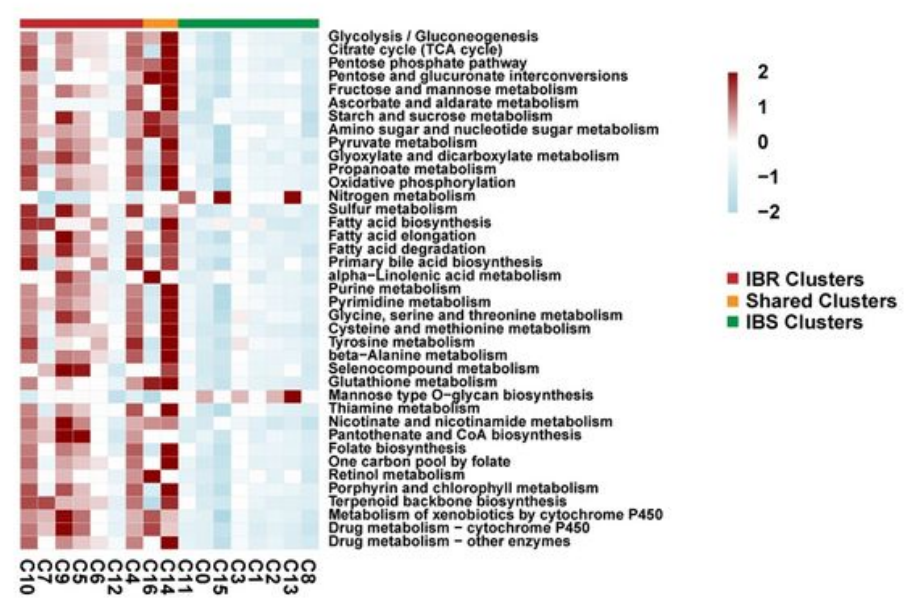

G

$\mathbf{F}$
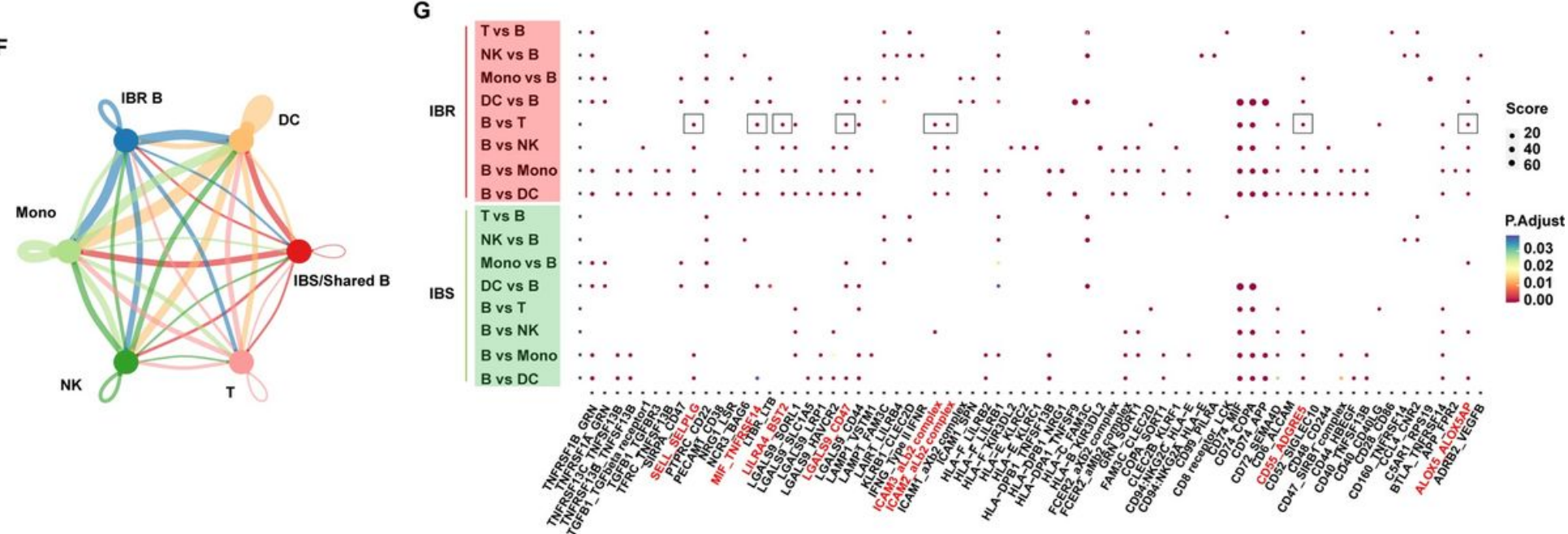

Figure 2

Distinct transcriptional pattern of ibrutinib-resistant (IBR) B cells and intercellular interactions among multiple cell types. A Left: unsupervised clustering of B cells showed in t-SNE plot. Right: t-SNE plot displays B cells from different types of samples. Red dots represent B cells from IBR patients; green dots represent $B$ cells from ibrutinib-sensitive (IBS) patients; blue dots represent $B$ cells from normal donors. $B$ Stem score visualization for B cell in the t-SNE plot. C Comparison of the stem scores of B cells from IBR patients, IBS patients and normal donors. Significance was calculated using the Wilcoxon rank-sum test. D Upper: proportion of cells derived from three sample types in each cluster. Red represents IBR patients. Green represents IBS patients. Blue represents normal donors. The dashed lines represent 0.3 and 0.5 , respectively. Lower: bar plot shows the number of cells in each cluster. The dashed line represents 200 . E Metabolic pathway enrichment in each cluster. Red represents IBR clusters. Green represents IBS clusters. 
Orange represents shared clusters. F Graph showing interactions among different cell types. Line thickness represents the number of significant interactions. G Graph displaying the summary of selected ligand-receptor interactions between different cell types. Circle size represents $p$-values (permutation test). Color gradient indicates the score of interaction.

A

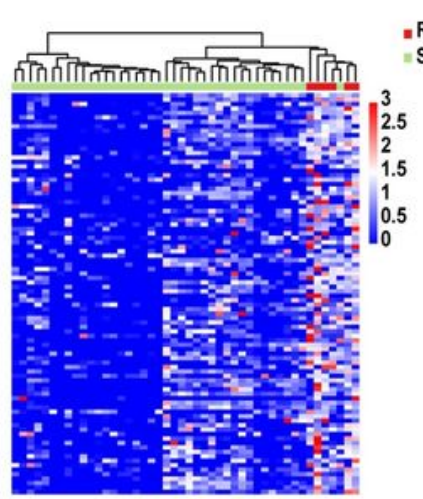

C

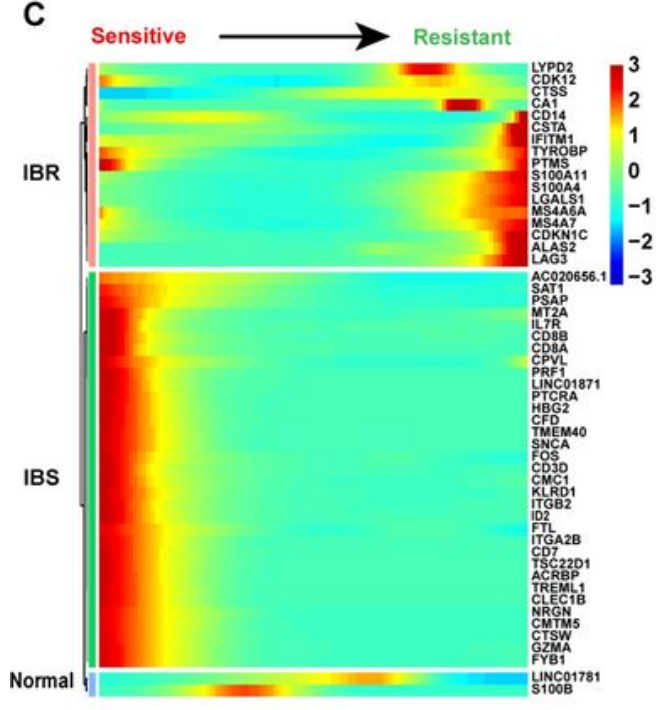

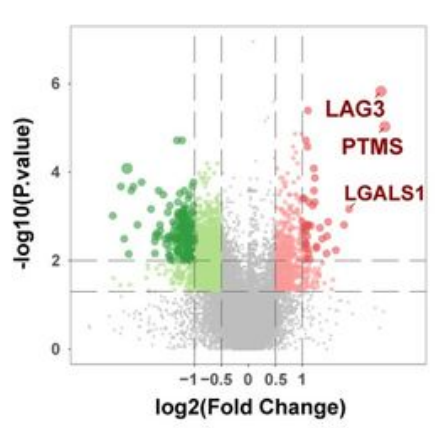

D

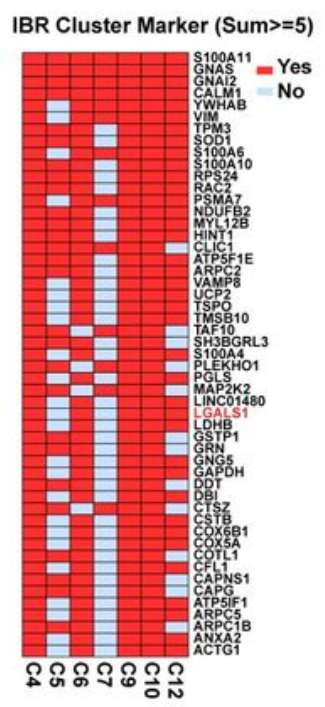

B

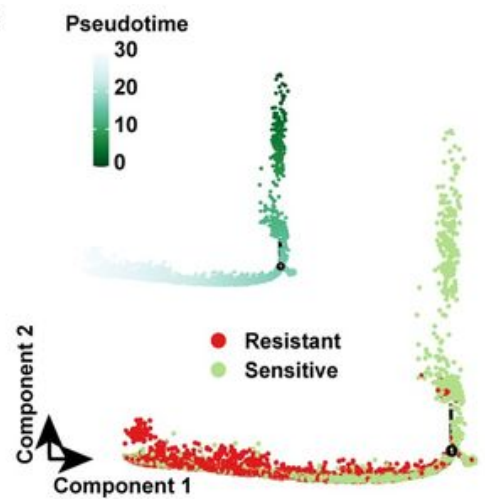

E

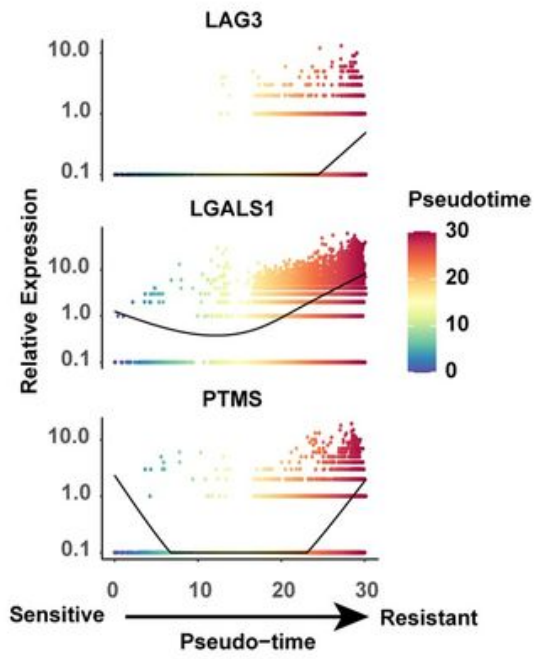

F

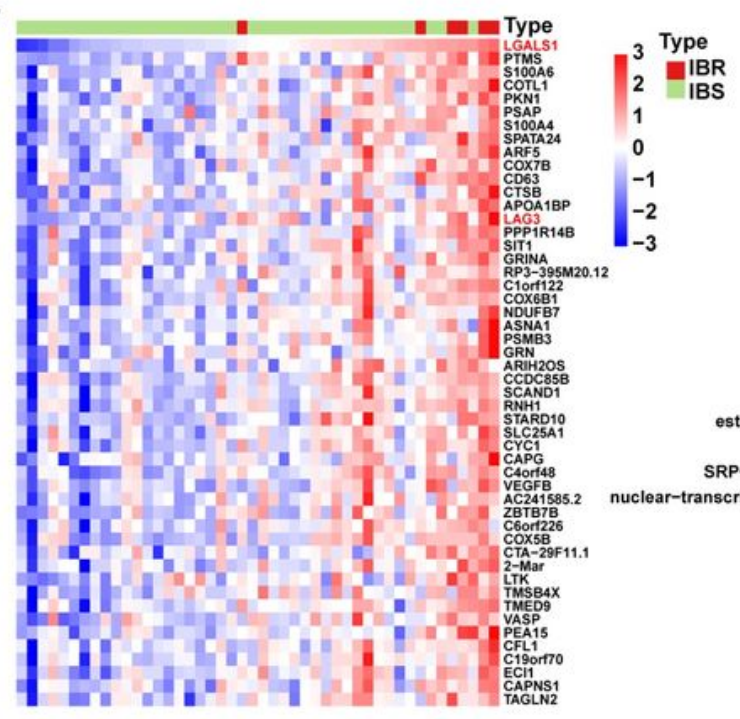

G

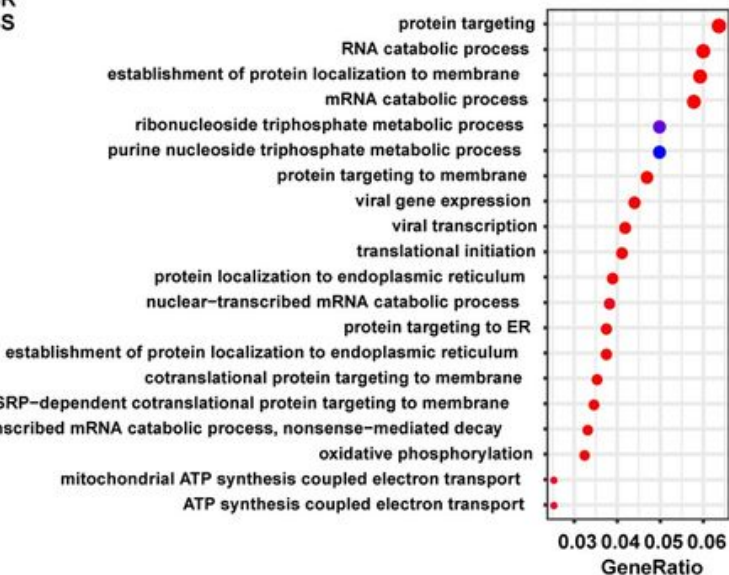

Count

: 40

-60
-70

P.adjust

$5.0 \mathrm{e}-13$

$1.0 \mathrm{e}-12$

$2.0 \mathrm{e}-12$

Figure 3 
Identification of key regulators of ibrutinib resistance based on bulk and single cell transcriptomic data. A Left: heatmap representing the differential gene expression between ibrutinib-resistant (IBR) and ibrutinibsensitive (IBS) patients based on bulk RNA-seq data. Red indicates IBR patients. Green represents IBS patients. Right: volcano plot depicting differentially expressed genes (DEGs). The significant up-regulated genes in IBR patients are shown with red, and the significant down-regulated genes in IBR patients are shown with green. B Developmental trajectory from IBS to IBR B cells based on scRNA-seq data. C Dynamic changes in gene expression from IBS to IBR. D Graph showing up-regulated genes in single-cell IBR clusters. E Graph showing dynamic changes in LAG3, LGALS1, and PTMS expression along the developmental trajectory from IBS to IBR. Color gradient indicates pseudo-time scores. F Heatmap visualization of genes co-expressed with LGALS1 based on bulk RNA-seq data. Patients were sorted by the expression of LGALS1. G Functional enrichment analysis of genes co-expressed with LGALS1. Dot size represents the number of enriched genes in the pathway. Color gradient represents the adjusted $p$ value. 

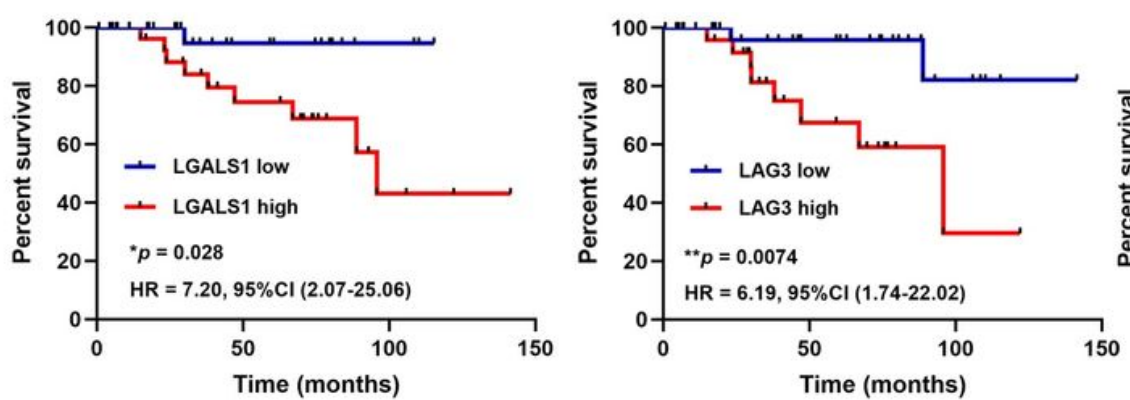

1 LGALS1 and LAG3 high

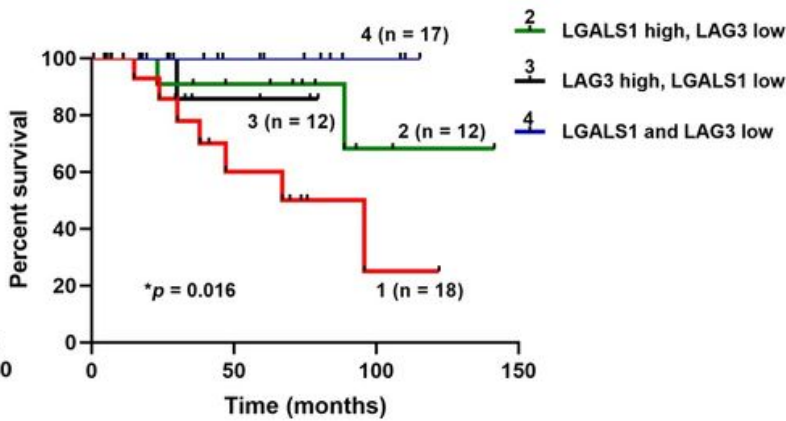

B

Survival for LGALS1

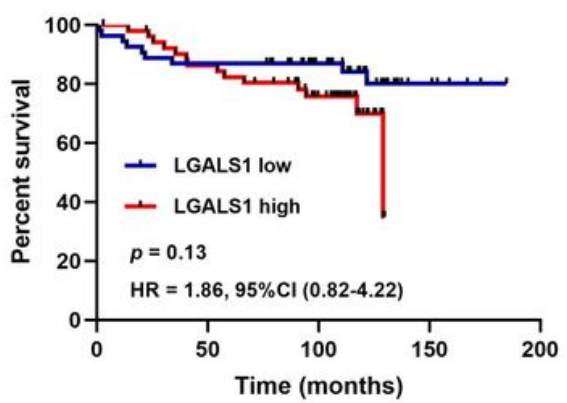

Survival for LAG3

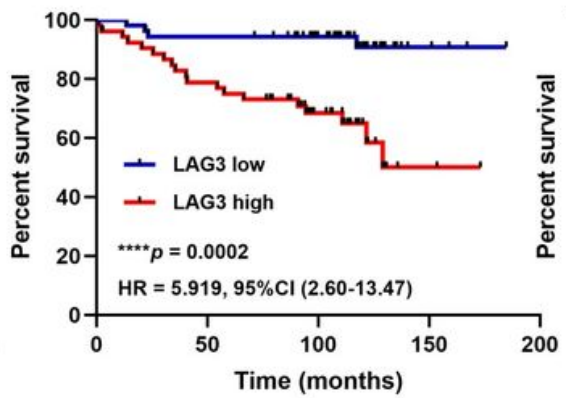

Survival for LGALS1+ LAG3

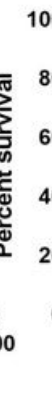

1 LGALS1 and LAG3 high 2. LGALS1 high, LAG3 low 3. LAG3 high, LGALS1 low 4 LGALS1 and LAG3 low

C

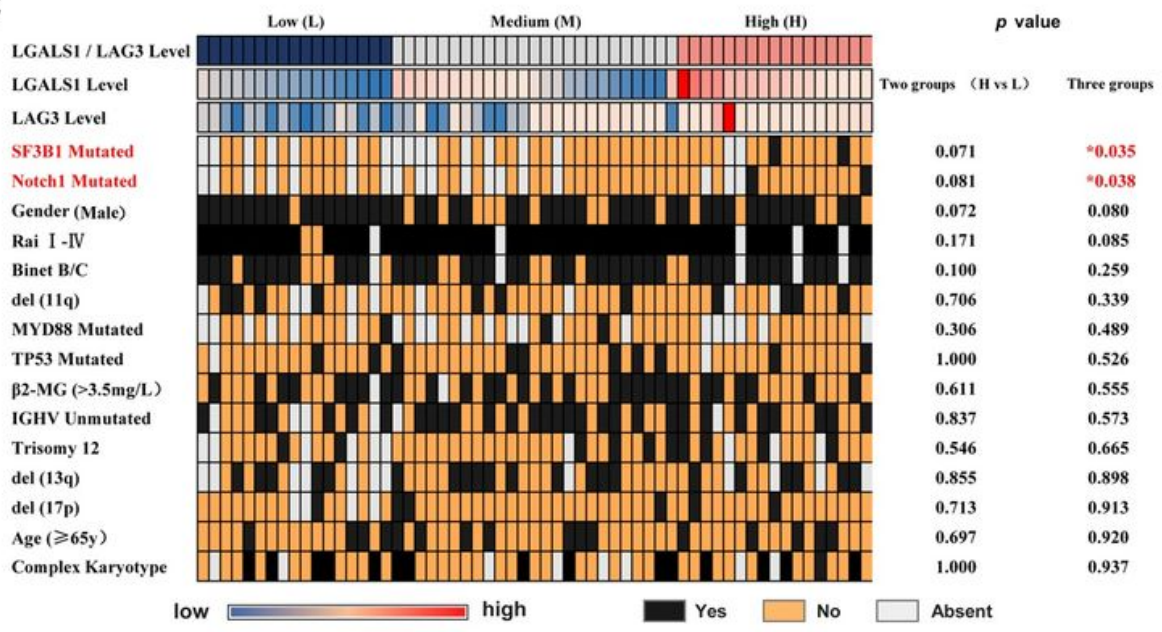

D

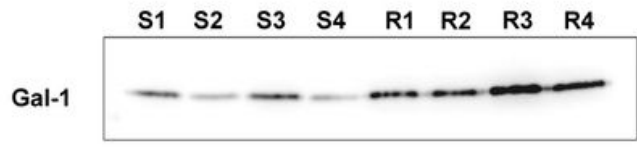

LAG3

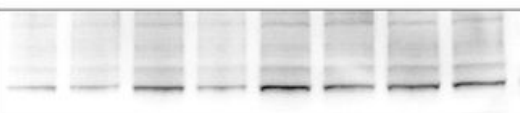

.573

.913

$\beta$-Actin

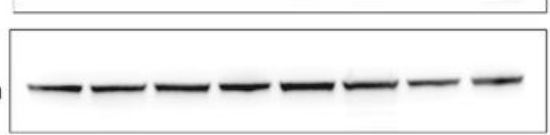

\section{Figure 4}

Increased LGALS1 and LAG3 expression correlates with poor survival in chronic lymphocytic leukemia (CLL). A Kaplan-Meier analysis of CLL patient samples based on LGALS1 and LAG3 expression $(n=59)$. ${ }^{*} p \leq 0.05,{ }^{*} p \leq 0.01$. B Survival analysis of patients from GSE22762 stratified based on LGALS1 and LAG3 expression. C LGALS1 and LAG3 mRNA levels in CLL patients. Schematic diagram of diverse clinical features and prognostic patterns in CLL samples. $\beta 2-M G, \beta 2$-microglobulin; del, deletion; IGHV, immunoglobulin heavy variable gene. D Gal-1 and LAG3 protein levels in four ibrutinib-sensitive (IBS) CLL patients and four ibrutinib-resistant (IBR) CLL patients. S, sensitive; $R$, resistant. 


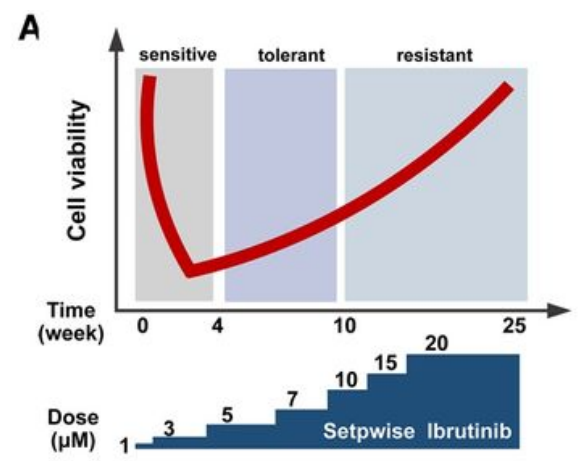

B

D

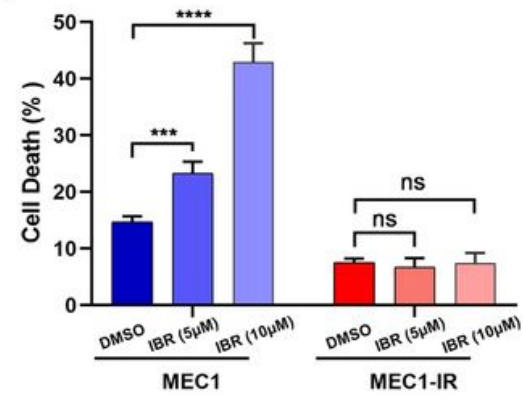

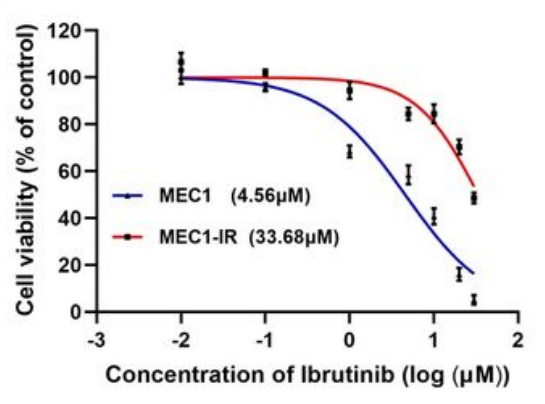

E

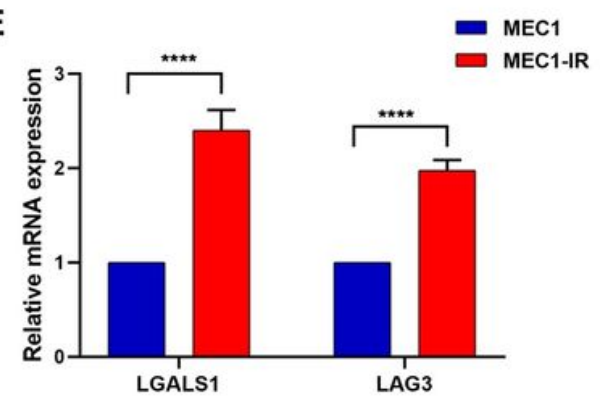

C

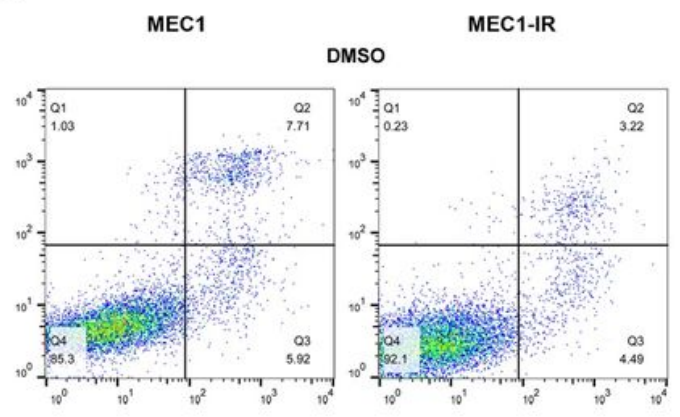

F

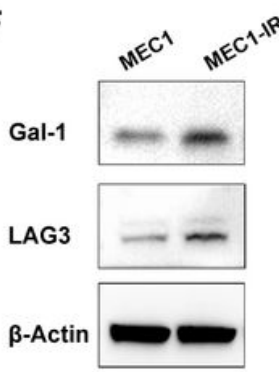

G

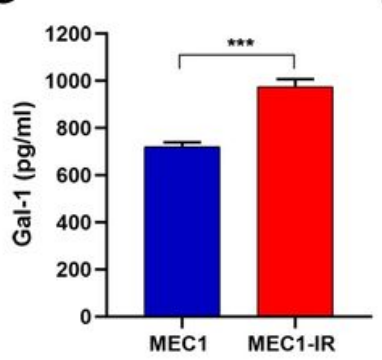

H

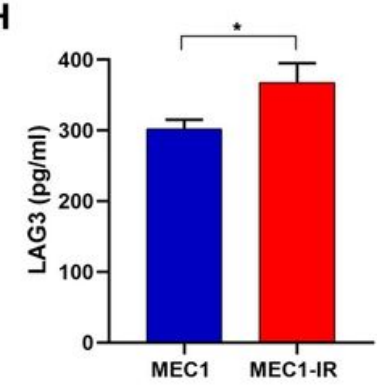

Ibrutinib $(5 \mu \mathrm{M}$

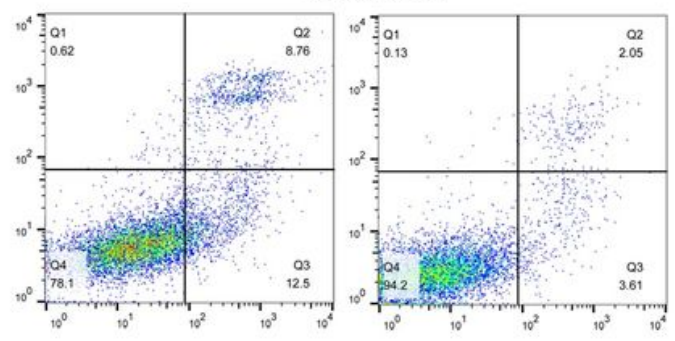

Ibrutinib $(10 \mu \mathrm{M})$

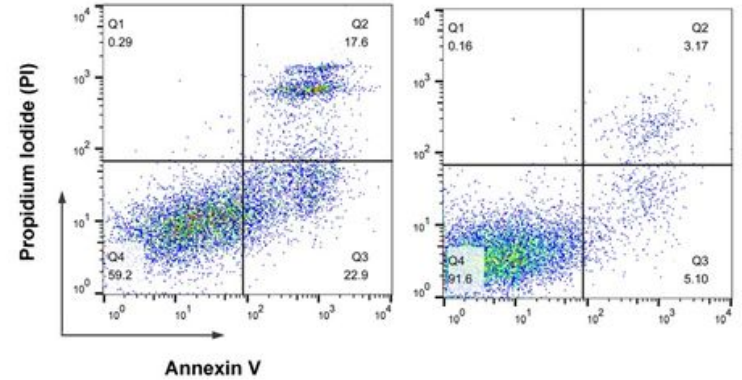

Figure 5

High LGALS1 and LAG3 expression in cells with acquired ibrutinib resistance. A Schematic diagram of ibrutinib-resistant chronic lymphocytic leukemia (CLL) cell line (MEC1-IR) establishment and cell viability throughout. B Relative proliferation of MEC1 and MEC1-IR cells treated with different ibrutinib concentrations for $48 \mathrm{~h}$. IC50 values were calculated from dose-response curves for four biologicallyindependent samples. The IC50 for each cell line is indicated in parentheses. C, D MEC1 and MEC1-IR cell apoptosis following exposure to ibrutinib (ibrutinib-resistant; IBR) for $48 \mathrm{~h}$. E-H LGALS1 and LAG3 RNA and protein levels in MEC1 and MEC1-IR cells estimated using qRT-PCR (E), western blotting (F), and $\operatorname{ELISA}(\mathrm{G}, \mathrm{H}) .{ }^{\star} \mathrm{p} \leq 0.05,{ }^{* \star} \mathrm{p} \leq 0.01,{ }^{* \star *} \mathrm{p} \leq 0.001,{ }^{\star \star * *} \mathrm{p} \leq 0.0001$. 
A MEC1

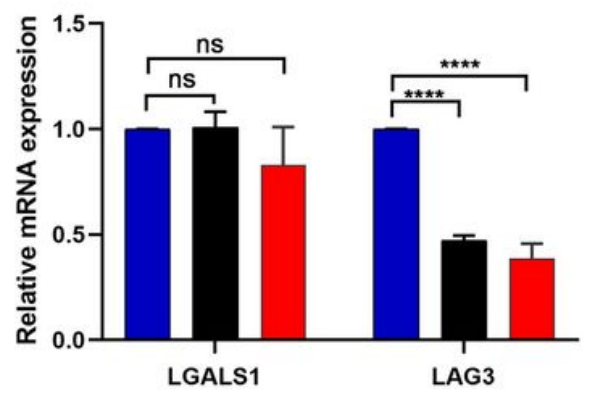

B

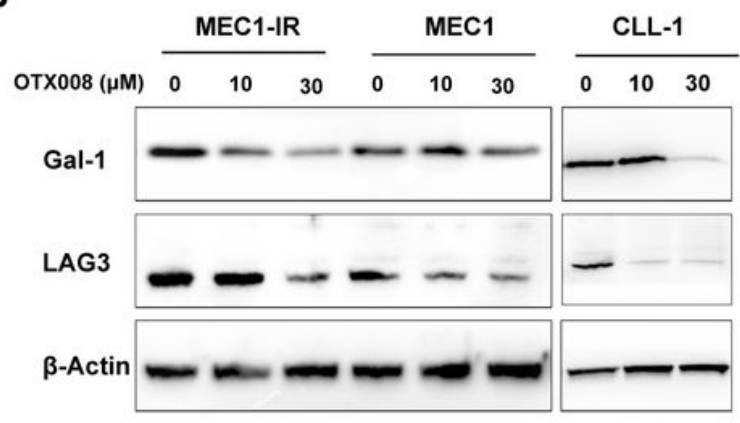

D

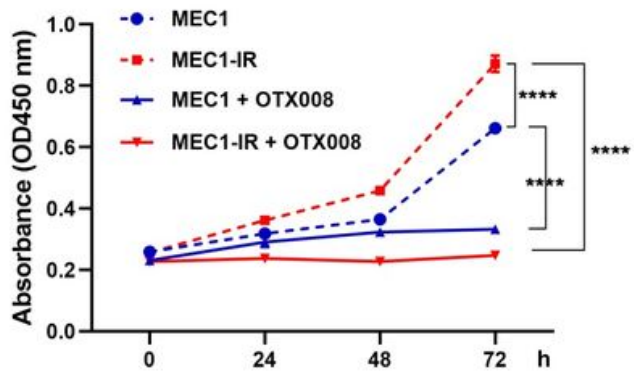

E

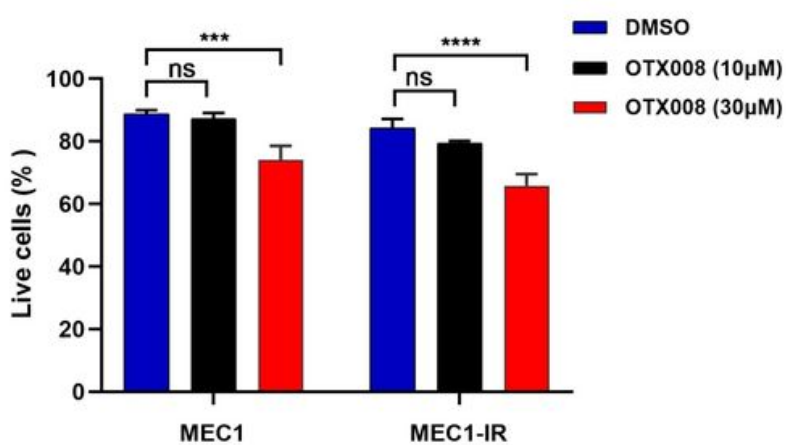

MEC1-IR

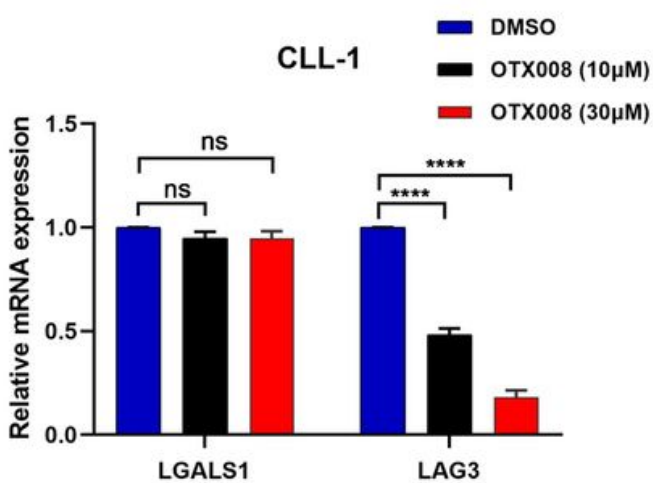

C

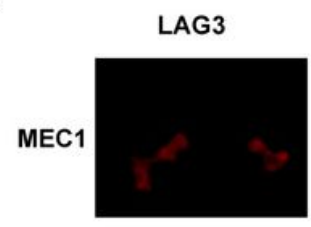

Gal-1

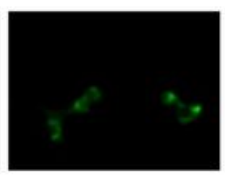

DAPI

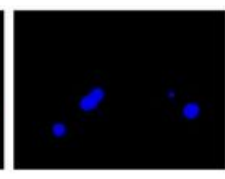

Merge
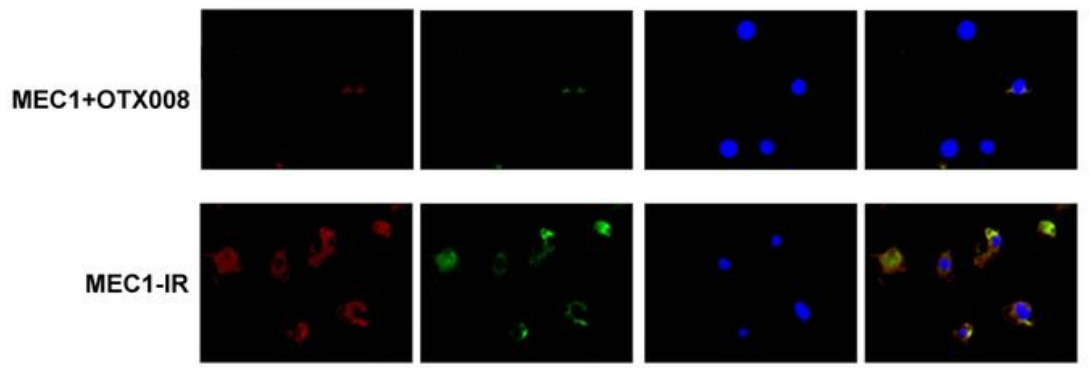

MEC1-IR+OTX008
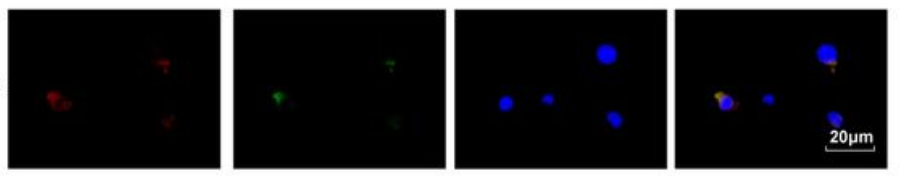

F

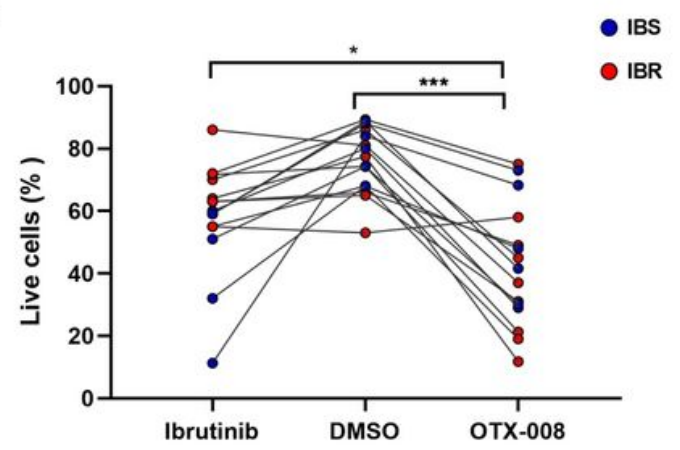

Figure 6

OTX008 inhibits CLL-cell growth and exhibits efficacy against IBR CLL cells. A Parental (MEC1) and acquired ibrutinib-resistant (MEC1-IR) CLL cell lines and primary CLL cells were treated with increasing OTX008 concentrations for $48 \mathrm{~h}$. Relative LGALS1 and LAG3 expression in CLL cells at the indicated OTX008 concentration was measured using qRT-PCR. B, C Western blotting and immunofluorescence analysis of Gal-1 and LAG3 in OTX008-treated CLL cells. D Cell proliferation was analyzed using CCK8 assays with or without OTX008 treatment. E Cell viability was analyzed using flow cytometry as the 
percentage of Annexin- $V$ and PI double-negative cells. F Freshly isolated PBMCs from CLL patients were incubated with $10 \mu \mathrm{M}$ ibrutinib and $30 \mu \mathrm{M}$ OTX008 for $48 \mathrm{~h}$. Cell viability was assessed using trypan blue staining. Cells with $>20 \%$ cytotoxicity at $10 \mu \mathrm{M}$ ibrutinib were regarded as IBS (blue, $n=6$ ) and those with $<20 \%$ were IBR (red, $n=9$ ). CLL, chronic lymphocytic leukemia; IBR, ibrutinib-resistant; IBS, ibrutinibsensitive.

\section{Supplementary Files}

This is a list of supplementary files associated with this preprint. Click to download.

- SupplementalData.docx

- SupplementalTable1.xIsx

- SupplementalTable2.xlsx

- SupplementalTable3.xlsx

- SupplementalTable4.xIsx

- SupplementalTable5.xlsx

- SupplementalTable6.xlsx

- SupplementalTable7.xlsx

- SupplementalTable8.xlsx

- SupplementalTable9.xlsx 